\title{
Parkinson's disease: a model dilemma
}

\section{The lack of a good animal model is frustrating efforts to curb disease progression, explains $\mathbf{M}$. Flint Beal.}

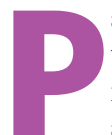

arkinson's disease is unique among the neurodegenerative disorders in that we understand a great deal about its circuitry and physiology, which are to a large extent caused by a loss of the dopaminergic neurons of the substantia nigra pars compacta. This knowledge has led to effective symptomatic treatment of the motor manifestations of the illness, initially with levodopa (L-DOPA) and then later with dopamine receptor agonists and deep brain stimulation.

Yet the efficacy of symptomatic therapy wanes after five to ten years, and patients develop increasing motor impairment, gait and postural difficulties, and cognitive dysfunction. The major aim now is to develop disease-modifying treatment. But if we are to slow or halt the underlying neurodegenerative process, we will need an even better understanding of the disease pathophysiology, embodied in better animal models, in which to test and develop new diseasemodifying therapies.

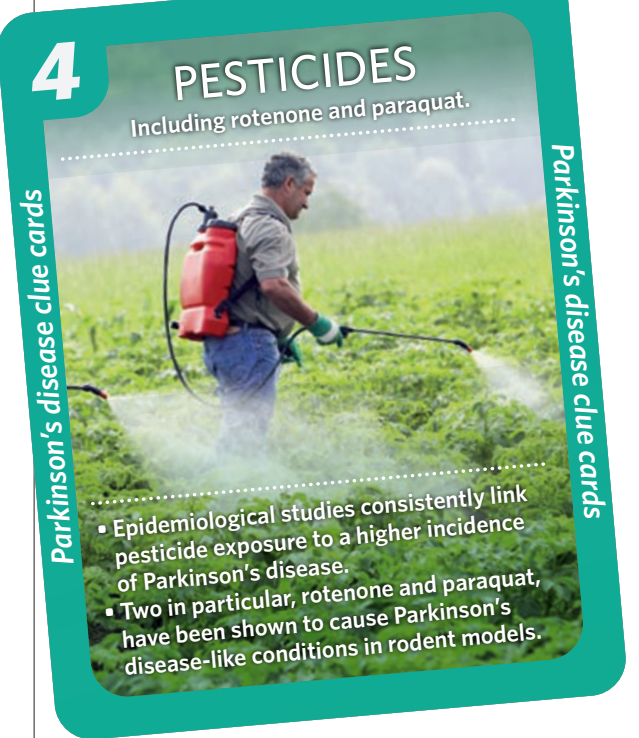

\section{The ideal animal model}

In order to be both a good model of human Parkinson's disease and a good test subject for new therapies, the ideal animal model should have a number of characteristic features. First, there should be a normal complement of dopaminergic neurons at birth, more than $50 \%$ of which are then selectively, gradually and measurably lost in adulthood. Second, the model should have motor deficits, including the cardinal symptoms of Parkinson's disease: bradykinesia, rigidity and resting tremor. Third, it should show the characteristic Lewy body neuropathology. Fourth, if the model is genetic, it should be based on a single mutation to allow robust propagation and facilitate crossing with enhancer or suppressor strains. Fifth, it should have a relatively short disease course of a few months, allowing rapid and less costly screening of therapeutic agents.

\section{Toxin-treated models}

Traditional animal models of Parkinson's disease are based on the use of toxins, which selectively accumulate in the substantia nigra dopaminergic neurons, causing cellular dysfunction and death.

6-hydroxydopamine (6-OHDA) is one such compound that produces substantia nigra degeneration in mice, rats, cats and primates. The standard approach is to use 6-OHDA to create a unilateral lesion in the brain, which is followed by administration of amphetamine. This causes the animal to start rotating 'away from' the lesion. This rotary behaviour can be reversed, for example by treatment with dopaminergic stem cells that restore normal circuitry.

The insecticide rotenone can also be used to create an animal model, as administration specifically inhibits complex I of the mitochondrial electron-transport chain. Betarbet and colleagues produced a model of Parkinson's disease by infusing rats intravenously with rotenone. The rats developed progressive degeneration of nigrostriatal neurons as well as cytoplasmic inclusions reminiscent of Lewy bodies. They also exhibited bradykinesia, postural instability and an unsteady gait, all of which improved after treatment with the dopamine agonist apomorphine. Other compounds that have been shown to produce selective degeneration of dopaminergic neurons include paraquat and trichloroethylene, both of which have been implicated in the causation of human Parkinson's disease. However, there is substantial variability in all of these models, which limits their usefulness for therapeutic development.

The best characterized toxin-based model of Parkinson's disease uses MPTP. The importance of this compound was discovered accidentally in 1982, when a group of young drug takers in California developed subacute onset of severe parkinsonism, caused by contamination of a synthetic opiate with MPTP. The administration of MPTP was subsequently shown to model Parkinson's disease in both mice and primates, by inhibiting part of the electron-transport chain in dopaminergic neurons of the substantia nigra. This leads to a reduction in ATP generation, but a more important effect might be increased freeradical production.

MPTP toxicity in primates replicates all of the cardinal clinical signs of Parkinson's disease. It has therefore proved useful for studying the striatal circuitry involved in Parkinson's disease pathophysiology. It causes a loss of locus coeruleus neurons, which are particularly vulnerable to the pathologic process in Parkinson's disease, whereas other neurons - such as those of the dorsal motor nucleus of the vagus and nucleus basalis of Meynert - are spared. Acute administration of MPTP does not cause Lewy body formation, although chronic administration produces synuclein inclusions. Overall, although a large number of therapeutic approaches have been tried in the MPTP model, its predictive value for humans has been inconsistent ${ }^{1}$.

Even though toxin-based models are convenient ways to quickly replicate much of the cellular dysfunction seen in Parkinson's disease, the extent to which they effectively and reproducibly mimic the entirety of the human condition is controversial.

\section{Genetic mouse models}

As an alternative to toxins, newer animal models have utilized genetic knockout and knock-in technologies - facilitated by a greater understanding of the genetic basis of Parkinson's disease.

One of the major drawbacks of the traditional models is the lack of Lewy body pathology. $a$-synuclein is a major structural moiety 
of Lewy bodies. Three point mutations in a-synuclein (A53T, A30P and E46K) are responsible for rare familial, autosomal dominant cases, and simple duplication or triplication of the $\alpha$-synuclein gene is sufficient to cause Parkinson's disease in humans.

Mice overexpressing wild-type $\alpha$-synuclein develop progressive accumulation of a-synuclein and ubiquitin-immunoreactive inclusions in the neocortex, hippocampus and substantia nigra ${ }^{2}$. The inclusions are composed of fine granular material, but lack the fibrillar aggregates that are characteristic of actual Lewy bodies. Dopaminergic neurons within the substantia nigra are spared, although there is a loss of dopaminergic terminals in the striatum.

Of the many a-synuclein mouse models, only A53T transgenics exhibit a full range of pathology including $\alpha$-synuclein aggregation, oligomers, fibrils, phosphorylation, ubiquitination and progressive age-dependent neurodegeneration ${ }^{3}$. These mice, however, do not show a loss of substantia nigra dopaminergic neurons, and motor deficits are caused by a loss of brain stem neurons and anterior horn motor neurons of the spinal cord.

The relationship between $\alpha$-synuclein and mitochondrial function in these mice is intriguing. A53T transgenics show mitochondrial abnormalities, including vacuolization and loss of cristae, as well as mitochondrial DNA damage. This is consistent with a number of other studies that have suggested that $\alpha$-synuclein might be imported into mitochondria, where it increases the production of reactive oxygen species and thereby damages DNA.

Mutations in the LRRK2 gene are another frequent cause of autosomal dominant Parkinson's disease. The most common mutation, G2019S, has frequencies of $1 \%$ in patients with sporadic Parkinson's disease and $4 \%$ in patients with the hereditary form. In some ethnic subgroups, such as North African Arabs and Ashkenazi Jews, mutations in $L R R K 2$ can be found in $30 \%$ or more of patients with Parkinson's disease.

Yet mouse models of LRRK2 mutations have so far failed to produce neurodegeneration of dopaminergic neurons ${ }^{4-6}$. Furthermore, an LRRK2-knockout mouse showed no increase in vulnerability to MPTP. My group at Weill Cornell together with neuroscientists from Columbia University recently characterized a mouse overexpressing the $L R R K 2^{R I 441 G}$ mutation, created using a bacterial artificial chromosome ${ }^{6}$. These mice developed age-dependent motor deficits leading to immobility and impaired rearing, and were responsive to L-DOPA and apomorphine.

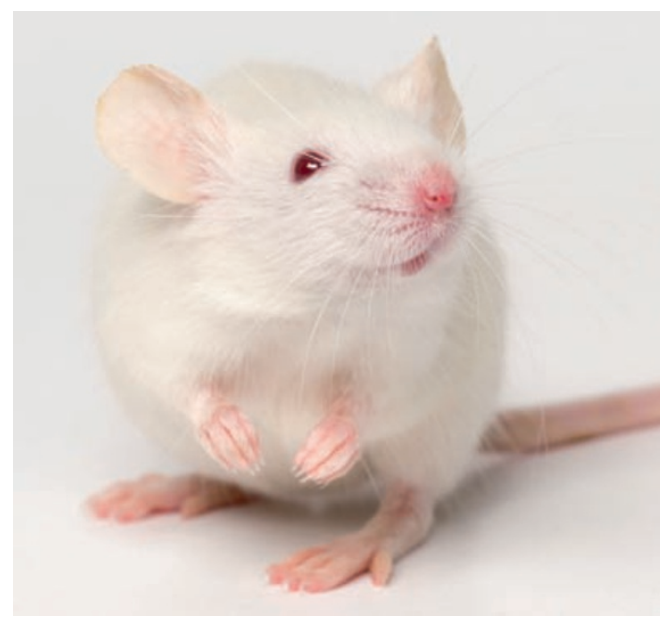

Mice are commonly used in Parkinson's disease research, particularly as genetic models.

In addition, there was impaired dopamine release in the striatum at ten weeks of age. Surprisingly, however, there was no loss of striatal dopamine or of dopaminergic neurons within the substantia nigra pars compacta. In contrast to the typical Lewy bodies, however, the major pathology observed in these mice was axonal spheroids, composed of phosphorylated tau, which were seen in both the striatal and the cortical regions.

Although these $L R R K 2^{R 1441 G}$ knock-in mice develop a robust behavioural phenotype, the lack of degeneration of dopaminergic neurons might limit how useful they will be in the development of therapeutic agents. The kinase domain of LRRK2, however, might prove to be a useful target for drug development, and could

\section{"One of the major drawbacks of the traditional models is the lack of Lewy body pathology."}

to progressive degeneration of dopaminergic neurons, supporting the idea that some parkin mutants might act in a dominant-negative fashion $^{7}$. These mutants therefore produce a better disease model than the knockout mice.

PINK1 encodes a serine threonine kinase domain with an amino-terminal mitochondrial-targeting motif. PINK1-knockout mice do not show any loss of dopaminergic neurons, and have normal levels of striatal dopamine and dopamine receptors.

DJ-1 is a redox-sensitive molecular chaperone with diverse functions. It upregulates redoxdependent kinase signalling pathways and helps to regulate antioxidant gene expression. Mutations in $D J-1$ cause a loss of chaperone function and instability. However, as with parkin and PINK1 knockouts, DJ-1-knockout mice do not potentially be of benefit for patients with either mutations of LRRK2 or sporadic Parkinson's disease.

Autosomal recessive Parkinson's disease features mutations in the parkin, PINK1 and $D J-1$ genes. Mutations in parkin were first identified in 1998 as the genetic cause of earlyonset parkinsonism in Japanese families, and they account for at least $20 \%$ of young-onset sporadic Parkinson's disease. Mutations in PINK1 are the second most common autosomal recessive mutation, contributing to between 1 and $7 \%$ of cases of early-onset Parkinson's disease. Mutations in $D J-1$ are a rarer cause.

Parkin-knockout mice show subtle abnormalities in both the dopaminergic nigrostriatal and the locus ceruleus noradrenergic systems. Some mutations of parkin appear to be autosomal dominant and, when produced by overexpression of mutant human parkin using a bacterial artificial chromosome transgenic model, lead exhibit any major abnormality, and the number of dopaminergic neurons and level of striatal dopamine are unchanged. $D J-1$-knockout mice do, however, show increased vulnerability to MPTP toxicity.

Unfortunately, with no loss of dopaminergic neurons, the parkin-, PINK1- and DJ-1-deficient mouse models have little utility outside of basic research and cannot be used to test neuroprotective agents.

\section{MitoPark mouse}

The most interesting, and perhaps most faithful, mouse model for Parkinson's disease developed to date ironically does not involve a known genetic mutation in the human condition. This is the MitoPark mouse model, produced by deleting the gene for the mitochondrial transcription factor Tfam, which plays a critical role in maintaining mitochondrial $\mathrm{DNA}^{8}$. 


\section{Personal experience}

In the 1990s, my group at Weill Cornell became interested in coenzyme Q10 for Parkinson's disease following reports of beneficial effects in some patients with known mitochondrial diseases, such as mitochondrial myopathy, encephalopathy, lactic acidosis and stroke-like episodes (MELAS) or myoclonic epilepsy associated with ragged-red fibres (MERRF). Coenzyme Q10 plays a role in the generation of ATP by mitochondria and it has antioxidant effects.

We carried out experiments that showed that coenzyme Q10 exerted neuroprotective effects against lesions produced by the mitochondrial toxins malonate, 3-nitropropionic acid and MPTP. When we initially tested coenzyme Q10, the MPTP model of Parkinson's disease was the best one available. The model, however, was produced by acute administration of MPTP over a few hours, and it did not produce Lewy bodies. Only more recently have we been able to show that coenzyme Q10 protects in a chronic MPTP model, in which the toxin is administered over one month.

The results of our initial studies were published in a series of papers between 1994 and 1998. In 2002, we completed a phase II trial in 80 patients, which showed efficacy. We are now nearing completion of enrolment for the phase III trial, with the expectation that we will have completed our studies and analysis in 2012. From start to finish, this process will have taken approximately 18 years.

This shows the difficulties in developing new therapies for neuroprotection. Eventually, efficacy in animal models needs to be confirmed in human clinical trials, which in turn serve to validate the usefulness and predictability of the model. Similarly, with genetic models such as $\alpha$-synuclein and LRRK 2 mice, it will be necessary to show that compounds that are efficacious in these models produce therapeutic effects in clinical trials.

The MitoPark mouse model experiences a progressive loss of midbrain dopaminergic neurons, and develops intraneuronal cytoplasmic inclusions containing mitochondrial protein and membrane components - although not $\alpha$-synuclein.
It shows decreased locomotion and reduced exploratory behaviour at 14 to 15 weeks of age, which progresses to limb rigidity at approximately 20 weeks, with sacrifice necessary at 45 weeks due to poor general health. Administering L-DOPA markedly improves motor performance, indicating that it shares many similarities with human Parkinson's disease.

Interestingly, this model shows that a primary deficiency of respiratory chain function in midbrain dopaminergic neurons leads to a progressive development of key Parkinson's disease features, including adult onset of neurodegeneration, a slowly progressive clinical course, formation of intraneuronal inclusions, cell death in the substantia nigra rather than in the ventral tegmental area and responsiveness to L-DOPA therapy. Yet without being based on a human genetic mutation, it is also of limited use for therapeutic development.

\section{Resolving uncertainties}

It remains unclear why there is no meaningful degeneration of dopaminergic neurons in any of the major genetic mouse models of Parkinson's disease. There have been attempts to increase the robustness of the phenotype by, for example, crossing a-synuclein transgenics with parkin and DJ-1 knockouts, and overexpressing a-synuclein with dopamine-specific promoters. Overexpression of LRRK2 can markedly exacerbate the $\alpha$-synuclein pathology in A53T a-synuclein mice, and LRRK2 knockout ameliorates the pathology induced by A53T $a$-synuclein ${ }^{9}$. The relationship between $\alpha$-synuclein and LRRK2 therefore remains of great interest.

Knocking out all three genes - parkin, PINK1 and DJ-1 - in a mouse model has negligible effect on dopaminergic neurons beyond that of the individual knockouts. This might be due to the genetic 'background', which is known to markedly modulate MPTP toxicity, and might play a role in the ability of dopaminergic neurons to resist degeneration. It will therefore be of interest to examine the effects of these genetic mutations with different genetic backgrounds.

Another line of enquiry concerns the effects of increased glutathione levels in parkin knockouts and increased glutathione peroxidase in $D J-1$ knockouts. However, knockout models of human neurologic diseases have frequently been unsuccessful, and as a general rule overexpression of dominant genes has a more consistent record of success.

\section{Future directions}

The development of animal models that truly recapitulate the age-dependent parkinsonian phenotype, $a$-synuclein-inclusion pathology and pathophysiology of Parkinson's disease would be of tremendous utility (see sidebar, Personal experience). The chemical toxin models utilizing 6-OHDA, rotenone and MPTP have been extremely useful for the development of symptomatic therapies, but their utility for the development of neuroprotective compounds and regeneration is unproven. Current transgenic mouse models have disappointed, lacking, as they do, a robust behavioural phenotype, degeneration of nigrostriatal dopaminergic neurons and Lewy bodies.

Parkinsonian non-motor symptoms are also deficient in these models as they fail to reflect olfactory dysfunction, constipation, depression and cognitive impairments. Many authors are putting increasing emphasis on non-motor symptoms, as they are particularly difficult to treat yet contribute greatly to the eventual disability of patients with Parkinson's disease. Dementia, for instance, commonly accompanies Parkinson's disease, and is known to be related to $\alpha$-synuclein and tau pathology, both of which also merit further investigation and have been identified as Parkinson's disease-associated genes in genomewide association studies.

The ultimate goal of current research into Parkinson's disease treatments is to develop novel therapeutic interventions that either slow or halt the degenerative process. For this to occur, it is vital to have an animal model that truly recapitulates the behavioural phenotype, neuropathology and pathophysiology of Parkinson's disease. Current animal models have so far failed to show validity in these areas, severely hampering attempts to develop new treatments. As such, creating an improved animal model is an ongoing frustration, and a major therapeutic, clinical and research need.

M. Flint Beal is Anne Parrish Titzell Professor and chairman of the Department of Neurology and Neuroscience at Weill Medical College, and director of the neurology service at the New York Presbyterian Cornell Campus.

1. Beal, M. F. Nature Rev. Neurosci. 2, 325-334 (2001). 2. Masliah, E. et al. Science 287, 1265-1269 (2000).

3. Lee, M. K. et al. Proc. Natl Acad. Sci. USA 99, 8968-8973 (2002).

4. Tong, Y. et al. Proc. Natl Acad. Sci. USA 106, 14622-14627 (2009).

5. Li, X. et al. J. Neurosci. 30, 1788-1797 (2010).

6. Li, Y. et al. Nature Neurosci. 12, 826-828 (2009)

7. Lu, X. H. et al. J. Neurosci. 29, 1962-1976 (2009).

8. Ekstrand, M. I. et al. Proc. Natl Acad. Sci. USA 104, 13251330 (2007).

9. Lin, X. et al. Neuron 64, 807-827 (2009). 